\title{
An analysis of stand-alone GPS quality and simulated GNSS quality for road pricing
}

\author{
M. Zabic \& O. A. Nielsen \\ Centre for Traffic and Transport, \\ Technical University of Denmark, Denmark
}

\begin{abstract}
Use of GPS for road pricing has often been suggested as the way of creating more efficient charging strategies than existing systems based on cordon lines or time use. In Denmark, Copenhagen participated with the AKTA project in the PRoGRESS programme, sponsored by the EU. The major part of the AKTA project was to equip 500 cars with GPS receivers. The paper presents the methods and results from a study of GPS quality in relation to road pricing in a dense urban area. The collected data from 500 cars over a two-year period in the Copenhagen region was analyzed in order to determine whether the stand alone GPS quality and reliability is adequate for implementation of an operational road pricing system in Copenhagen. The results from the analysis show that the satellite availability in Copenhagen is not sufficient to form the basis for a reliable operational road pricing system. The narrow street canyons in downtown Copenhagen prevent a sufficient amount of satellite signals to reach street level, which causes too many gaps in the position logs to implement a fair and reliable taxation scheme. The need for augmentation is evident, and with the advent of Galileo within a few years, it is relevant to investigate how integrated GPS/Galileo receivers will change the situation. With a Galileo simulator, an analysis of the GNSS (Global Navigation Satellite Systems) availability in the streets of Copenhagen was carried out. The results show, that there will still be streets in downtown Copenhagen were the receiver-satellite geometry represented by the HDOP is not sufficient for reliable positioning during the full 24 hours of a day, even when combined GPS-Galileo receivers are introduced. Keywords: GPS, road pricing, GNSS, GIS, Galileo simulation, urban transport.
\end{abstract}




\section{Introduction}

Road pricing is one among several approaches for reducing congestion on the streets in larger cities. As described below, GPS based road pricing is considered one of the best approaches for some cities. Drivers can be charged based on where, when, and how much they drive in the streets. However, for a GPS based system to work it must be reliable, and that can be a problem in the city centres in older cities, where the streets are narrow and the buildings are tall. A sufficient number of GPS signals might not reach the receivers in the vehicles causing lack of position registrations, and in the end problems with the charging schemes.

The AKTA experiment, as described in the following section, was carried out in 2001-2003, where 500 cars were equipped with GPS receivers. The GPS data was logged when the vehicles were in motion, and a very large and complete data set was collected. The GPS data has formed the basis for a throughout analysis of the GPS availability in Copenhagen, and the results of this analysis are given in the following. The analysis shows that there are streets in Copenhagen where GPS based road pricing are not feasible because of limited satellite availability. Therefore, the idea of developing software for analyses of simulated Galileo satellite availability was born.

This lead to a number of analyses where the satellite availability in some streets in Copenhagen were analysed for simulated situations with GPS only, Galileo only and a combined satellite constellation with both GPS and Galileo satellites. Therefore the paper covers two different approaches of analysing GNSS satellite availability; one approach is based on real GPS data, and the other is based on simulations. First a general introduction to the field experiment, before we look at the analyses.

\subsection{An introduction to the experiment}

The Copenhagen AKTA-experiment (the Danish abbreviation for alternative driving and congestion charging) tested such a system on 500 cars over a twoyear period [3]. AKTA tested a km.-based rush hour charging scheme, a scheme with all day charges (but double charge in the rush hours) and a scheme with cordons (refer to figure 1)

GPS data were colleted for 500 cars over a period of 2 years. The database includes approx. 250,000 trips and 120,000,000 GPS observations. The GPS receivers calculated and stored the car's position every second. For each car, different information was registered by the GPS receiver for every trip made. The most important information, besides time and date, is the $\mathrm{X}$ coordinate, the Y coordinate, the number of visible satellites, HDOP (Horizontal Dilution of Precision), distance and speed. The united database with this information was imported into ArcGIS, where different types of analyses were performed on the data.

The accuracy of every GPS based observation depends on the number of satellites in view, the quality of each signal (HDOP) and the direction the satellites are located by in respect to the vehicle and its movement. The receiver 
needs minimum 4 satellites in view to estimate $\mathrm{x}, \mathrm{y}$ and $\mathrm{z}$ as well as the time, which is used to estimate the position of the satellites. A HDOP value less than 4 indicates a good signal quality and hence a good measurement. Usually, reliable uninterrupted positioning can only be realized under an open sky or with minimum obstructions. However, GPS must provide sufficient availability and reliable service for road pricing even if the performance of the existing system may decrease under difficult operational conditions. The signal reception is therefore specifically sensitive in built-up areas given that the signal problems are often caused by high buildings that cut off the signals. These signal problems are also seen in wooded areas and while driving through tunnels and under bridges.

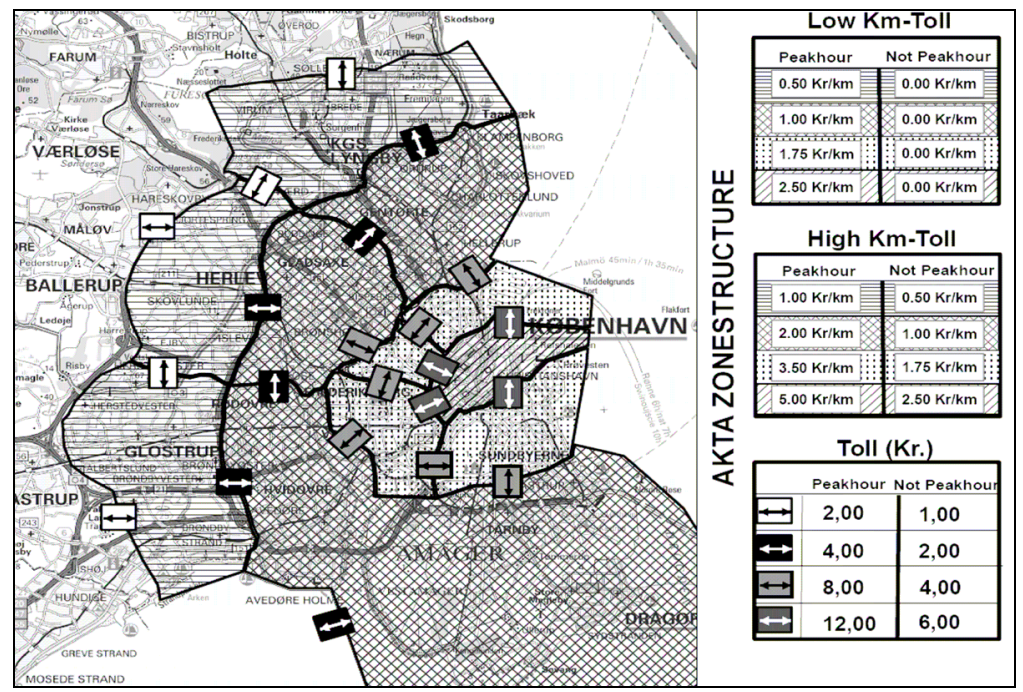

Figure 1: Different pricing schemes in AKTA (Background map from KMS, Copyright Kort and Matrikelstyrelsen G X-03, www.kms.dk).

\section{Availability of GPS in Copenhagen, field tests}

The suitability of the streets for road pricing was examined based on average values calculated for every street in downtown Copenhagen. In connection with road pricing it was interesting to examine which streets were suitable with regard to the satellite conditions.

One street may have good satellite conditions in one end, while the conditions worsen in the opposite end, due to transverse streets or other changes in the surroundings. The satellite conditions were examined for the road network in Copenhagen by establishing a buffer (of $30 \mathrm{~m}$ ) around every street in the network. Afterwards, the average GPS data (here the average satellite number and the average HDOP) were calculated for every street section based on the data within the buffer. 
The results revealed a correlation between the GPS quality and the density of the built-up areas in Copenhagen. The GPS availability is worse in the neighbourhoods in the city centre, where critical values were found (Figure 2A). Better signals appeared within the parks in Copenhagen given that the critical values decrease in these areas.

The GPS points are not directly related to a digital roadmap. In many cases it might be ambiguous which road the point "belongs to". Therefore a mapmatching algorithm had to be created in order to relate the GPS points to the network. This algorithm is described by Nielsen and Jørgensen [4]. One of the advantages of map-matching is that sections of trips without GPS observations are estimated using the most efficient path principle. Therefore, it was not only possible to analyze the quality of the GPS observations, when there were sufficient observations to estimate a location, but also to analyze the occurrence of missing observations.
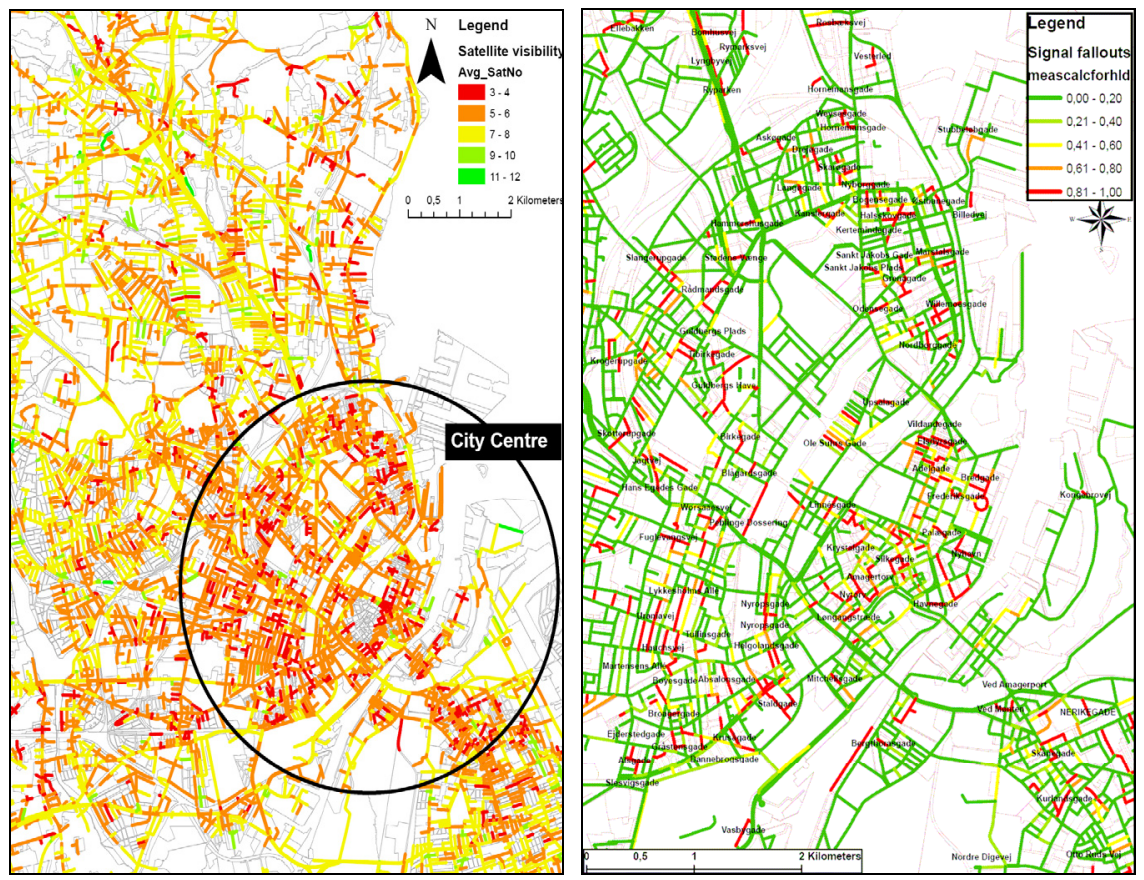

Figure 2: A (left): Average satellite visibility per street in Copenhagen (road map based on KRAKS kdv geodatabase, www.krak.dk). B (right): Signal fallouts scale per street in Copenhagen (road map based on KRAKS kdv geodatabase, www.krak.dk).

Since the original GPS data did not contain information about the actual occurred signal fall outs, due to the receiver being unable to determine the position with less than 3 visible satellites, it was found interesting to examine the map matched data, where these signal fall outs were registered and the position 
estimated. In the map matched database, all signal fall outs were registered for every street section. Based on that, a ratio between the number of actual signal fallouts and the total number of loggings in the street section were calculated for each street in Copenhagen. Hence the ratio value for the street section was 1, if all the loggings were signal fall outs, and 0 if there were no signal fall outs in that street section. The ratios were plotted on a map in a GIS, in order to show the scale of the signal fall-out problems. Figure 2B illustrates, with red, those streets in Copenhagen, where signal fall outs occurred in more than $80 \%$ of all positions. Furthermore, the results showed that the smaller side roads had more signal fall outs than the wider ones. The difference is quite systematic, since all the major (and therefore wider) roads have none or only a few signal fall outs.

If calculating the number of visible satellites and the HDOP for areas with different land use categories, then the relationship between the satellite visibility and the surroundings is emphasized. With five characteristic land use categories chosen in order to point out the most important differences, the average satellite number and HDOP for each of the 5 categories appear on figure 3.

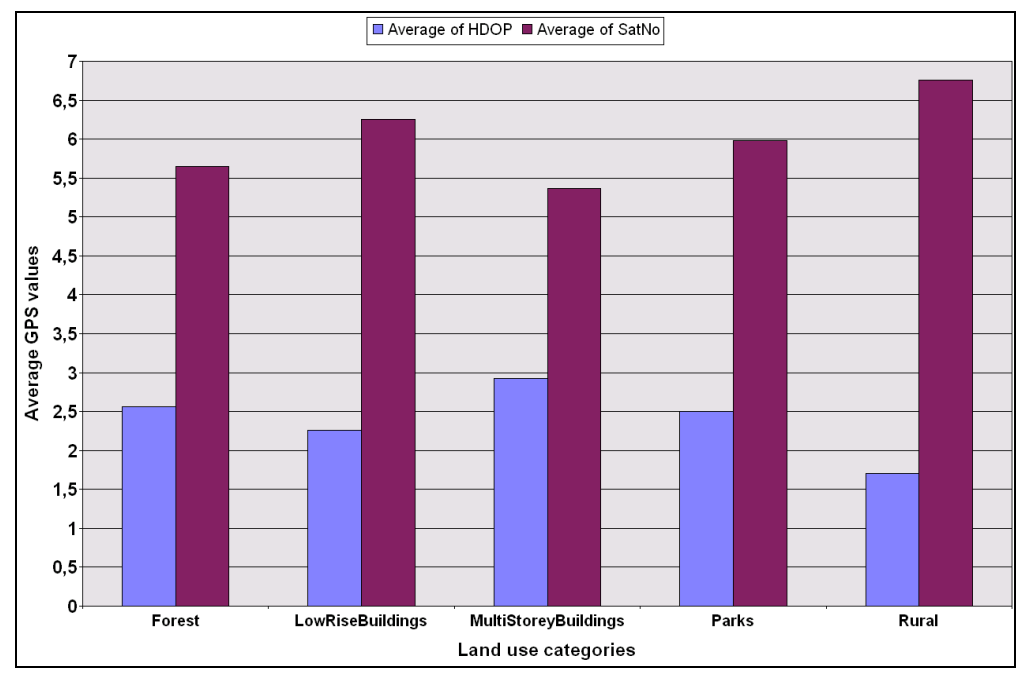

Figure 3: $\quad$ Satellite visibility and HDOP for different area types.

As it appears from the analysis [5], the GPS quality depends on the density of the built-up areas, which is why the problem areas are found in the most closely built-up neighbourhoods. Also the receiver satellite geometry was analysed. The HDOP was logged during the trips, and the worst HDOP based on data from the street segments was calculated. The results are shown in figure 4. Generally, a better geometry is available for street sections over bridges and along parks, and for the wider streets. The streets are 10-15 meters wide, the buildings are 5 or 6 stories high, and the HDOP is indicating that reliable GPS positioning is, on average, not possible in these streets. Based on these analyses the results show 
that GPS alone is not a sufficient mean for providing position information for a reliable road pricing system in Copenhagen.

\section{Availability of GNSS, simulations}

Will the situation be improved when the European Galileo satellite navigation system becomes available? In order to answer this question a Galileo simulator was developed based on a spatial 3D City Model (provided by the Danish company BlomInfo), a ray tracer (developed by Nielsen [2]) and a street network. The simulation of Galileo satellite positions was based on preliminary Keplerian elements provided by the Galileo Project Office (ESA) and included 27 satellites. For an in depth description of the Galileo simulator and the technical details refer to Jensen et al. [6]. The ray tracer was used for simulation of the path of the GNSS satellite signal from the position of a satellite to a given point in the model. Using the ray tracer and the $3 \mathrm{D}$ city model in combination, it was possible to estimate whether the signal will be blocked by buildings. All the features was combined in a software package called "The DOP simulator" and was handled through a graphical user interface which ran on a windows platform so that the software is useable with other city models provided in the same data format. The DOP simulator was run first for GPS only, then for Galileo only, and finally a combined GPS/Galileo simulation was carried out. A 24 hour test run was carried out, using the full 24 hours of GPS and Galileo satellite positions.

\subsection{Satellite visibility}

The average number of satellites visible for a location within the area tested is almost identical for GPS and Galileo. The standard deviation is slightly better for Galileo, but the difference is small. However, the average number of satellites visible is almost doubled by using combined GPS/Galileo receivers, a major improvement. Table 2 shows the combined mean and standard deviation of the number of visible satellites for all the streets analysed. Results of all the points are shown in the Table 2, and the statistics are only truly valid for this part of Copenhagen. However, the numbers give a very good indication of the improvement in satellite coverage that can be expected when the 27 satellite constellation of Galileo is available, and when combined GPS/Galileo receivers are used.

A closer look at the critical number of observations is given in Table 1. For a receiver to determine a position in $3 \mathrm{D}$, observations from at least four satellites are necessary. If ignoring the height, three observations are sufficient. A minimum requirement in connection with road pricing, where height information is normally not relevant, is thus at least three visible satellites for any time epoch, and for any given point on a street in the city.

The obtainable improvement by using combined GPS/Galileo receivers for positioning in downtown areas is clearly visible in Table 1. More than 4 satellites will be visible for $99 \%$ of the time in the streets used for this test using combined 
GPS/Galileo. Galileo alone provides a small improvement compared to GPS only. But it is with both systems fully operational that the real improvement is obtained, and the minimum number of visible satellites (at least 3) will be available basically all the time.

Table 1: $\quad$ Percentage of time epochs and locations with less than 3, 3, 4, and more than 4 visible satellites, 24 hour simulation.

\begin{tabular}{|l||l|l|l|l||}
\hline & $<3$ SVs & 3 SVs & 4 SVs & 4 SVs $<~$ \\
\hline \hline GPS & $2.3 \%$ & $7.7 \%$ & $11.0 \%$ & $79.0 \%$ \\
\hline Galileo & $2.4 \%$ & $6.0 \%$ & $10.0 \%$ & $81.6 \%$ \\
\hline $\begin{array}{l}\text { Galileo } \\
\text { /GPS }\end{array}$ & $0.0 \%$ & $0.1 \%$ & $0.8 \%$ & $99.1 \%$ \\
\hline
\end{tabular}

\subsection{Receiver-satellite geometry}

A sufficient number of visible satellites alone are not enough for an acceptable position solution. A good receiver-satellite geometry is also important, and the dilution of precision (DOP) is an easy mean for evaluating the geometry based on the satellite and receiver positions. In this case we have focused mainly on the horizontal HDOP, since the quality of the horizontal position is of most importance in connection with road pricing. However, also statistics of the $3 \mathrm{D}$ position DOP (the PDOP) are given in the table below.

Table 2: Mean and standard deviation of HDOP and number of visible satellites for GPS only, Galileo only, and combined GPS/Galileo, all based on a 24 hours simulation.

\begin{tabular}{|c|c|c|c|c|c|}
\hline & Mean & Std. dev. & & Mean & Std.dev. \\
\hline $\begin{array}{l}\text { GPS } \\
\text { HDOP } \\
\text { PDOP }\end{array}$ & $\begin{array}{l}2.6 \\
4.3 \\
\end{array}$ & $\begin{array}{l}5.0 \\
7.0 \\
\end{array}$ & $\begin{array}{l}\text { GPS } \\
\text { Number of SVs }\end{array}$ & 6.5 & 1.8 \\
\hline $\begin{array}{l}\text { Galileo } \\
\text { HDOP } \\
\text { PDOP }\end{array}$ & $\begin{array}{l}2.4 \\
3.9\end{array}$ & $\begin{array}{l}4.7 \\
6.2 \\
\end{array}$ & $\begin{array}{l}\text { Galileo } \\
\text { Number of SVs }\end{array}$ & 6.4 & 1.5 \\
\hline $\begin{array}{l}\text { Galileo/GPS } \\
\text { HDOP } \\
\text { PDOP }\end{array}$ & $\begin{array}{l}1.5 \\
2.3\end{array}$ & $\begin{array}{l}2.6 \\
3.3\end{array}$ & $\begin{array}{l}\text { Galileo/GPS } \\
\text { Number of SVs }\end{array}$ & 12.3 & 3.4 \\
\hline
\end{tabular}

Again, these numbers are determined based on data from all streets in the area analysed, and they are thus specific for the given part of Copenhagen. But the statistics can be used as a general indication of the improvement obtainable with Galileo. Galileo performs slightly better than GPS, and the improvement to be achieved with combined GPS/Galileo receivers is again significant. For the HDOP the improvement with combined receivers is about $37 \%$ compared to GPS only, and about $29 \%$ compared to Galileo only. To illustrate the simulated 
spatial variation in HDOP values we refer to figures 4 and 5 where the minimum HDOP value through the 24 hours is shown for the streets of the model.
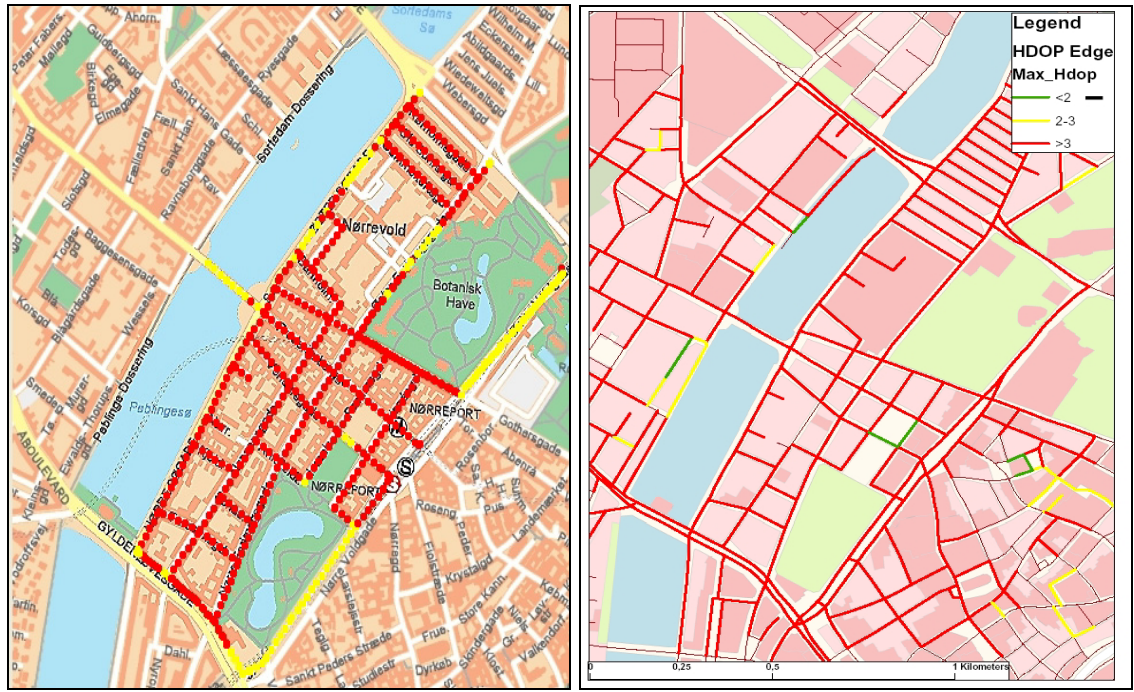

Figure 4: Left: Worst HDOP during 24 hours, GPS only. Green: HDOP $\leq 2$, Yellow: $2<$ HDOP $\leq 3$ and Red: HDOP $>3$. Right: Worst HDOP logged by GPS receivers collecting data in the streets during the AKTA experiment (road map based on KRAKS kdv geodatabase, www.krak.dk).
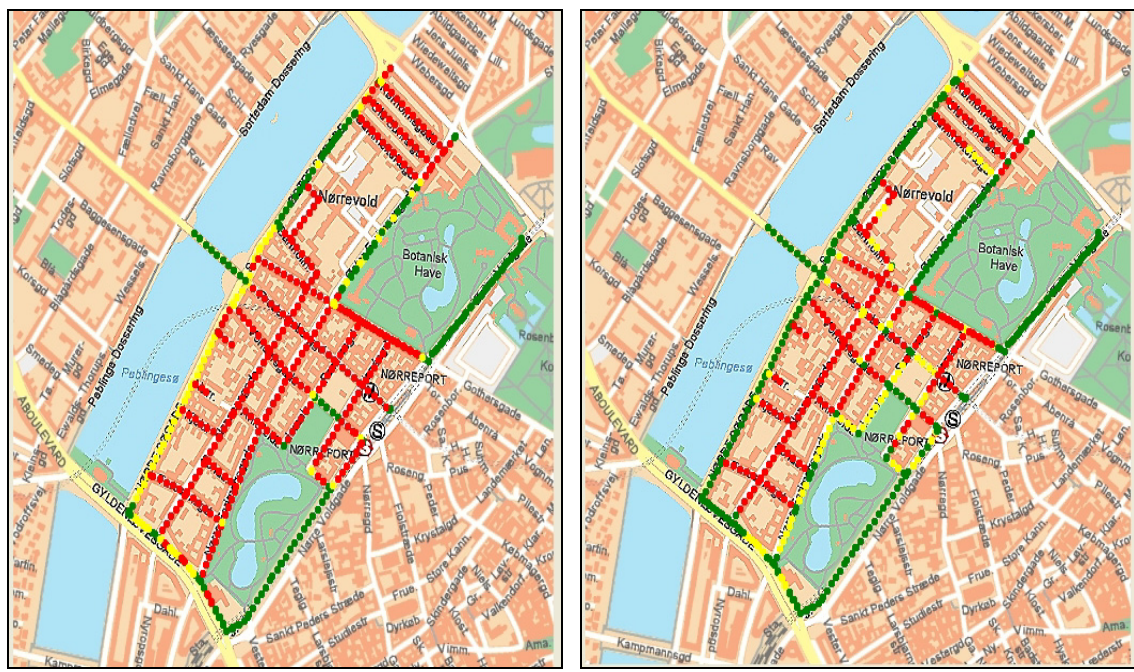

Figure 5: Left: Worst HDOP during 24 hours, Galileo only. Colour scale given in figure 4. Right: Worst HDOP during 24 hours, Combined GPS and Galileo. Colour scale given in figure 4. 
The scaling is based on our own experience with GPS positioning. The position accuracy is generally good when the HDOP is smaller than 2 . Theoretically, according to Misra and Enge [1], the 99 percentile of the HDOP is 1.6 considering the full GPS constellation on a global basis, and no obstructions.

The satellite coverage is generally best for the wider streets, and for streets running along parks and lakes. The improvement by using combined GPS/Galileo receivers is easily seen with this type of illustration. Some streets with poor satellite geometry with GPS or Galileo only, do reach an acceptable HDOP with combined GPS/Galileo. For many of the narrow streets the situation is, however, far from perfect even with a combination of GPS and Galileo.

The results of the simulations were at last compared with the analysis based on GPS data collected in situ during the AKTA project (Jensen et al. [6]), and the comparison showed that the results were identical, which verified that the simulation scenario is reasonable.

When summing up on the simulations carried out with the DOP Simulator software, it seems clear that the GNSS coverage in downtown Copenhagen is improved by the satellite constellation obtainable with a combined GPS/Galileo receiver. Still, however, there will be streets where reliable GNSS positions will be difficult to obtain 24 hours a day. In order to obtain a fully functional reliable road pricing system, the conventional GNSS positioning algorithms must be supplemented with a filter, which can be used for improving the positioning performance when driving through some of the narrow streets. With a good filter the satellite availability becomes slightly less important, since the position is determined based on previous positions and more advanced mathematical routines. Another mean for improving a GNSS based road pricing system can be the use of pseudolites mounted of carefully selected sites in the city. Pseudolites are transmitting GNSS like signals that can be used, just as real satellite data, to improve positioning performance.

\section{Conclusions and perspectives}

The tests described comprise an analysis of the availability of GNSS for road pricing. The AKTA experiment was described, and the results on GPS signal availability were discussed with respect to average satellite visibility, missing position information, and average HDOP. Based on the analyses it is concluded that GPS alone is not a sufficient mean for providing position information for a reliable road pricing system in Copenhagen.

Based on a 3D model of Copenhagen and a Galileo simulator, the theoretical availability of GNSS signals for a selected neighbourhood of Copenhagen was analysed for a GPS only, a Galileo only and a combined GPS/Galileo scenario. The simulations show that with combined GPS/Galileo the minimum number of satellite signals required, will be available continuously during the 24 hours for all streets tested. The HDOP is improved considerably by a combined GPS/Galileo solution compared to using one of the systems alone. But none of the scenarios tested showed a fully ideal geometric availability during the 24 hours used for the simulations. 
Whether a road pricing system can be implemented based on these results is mainly a political decision. However, there are several technical solutions to improve the position accuracy. Augmentation by filtering techniques, pseudolites, or integration with other sensors is some of the options.

Further analyses can be carried out using the DOP Simulator software. For the simulations carried out here, the GPS receiver was located at ground level, basically on the street. A slightly better performance is expected if the receiver is located at an altitude of 1 or 2 meters above the ground. This might be more realistic considering installation of GPS antennas on the roof of the vehicles.

\section{Acknowledgements}

Thanks to Anna B.O. Jensen, Helene M. Overø and Brian Ravn (The Technical University of Denmark) for developing the DOP simulator. BlomInfo and the City of Copenhagen are acknowledged for making parts of the $3 \mathrm{D}$ model of Copenhagen available. Galileo Project Office, ESA, is acknowledged for providing nominal preliminary Keplerian elements for the Galileo satellites and Morten Nielsen, BlomInfo, is acknowledged for making the raytracing code available.

\section{References}

[1] Misra, P. and Enge, P. Global Positioning System, Signals, Measurements, and Performance. Ganga-Jamuna Press. ISBN: 0-9709544-0-9.

[2] Nielsen, M. Ø. True orthophoto generation, M.Sc. thesis. Informatics and Mathematical Modelling, Technical University of Denmark.

[3] Nielsen, O. A. Behavioural responses to pricing schemes: Description of the Danish AKTA experiment. Journal of Intelligent Transportation Systems. Vol. 8(4). Pp. 233-251. Taylor \& Francis. 2004.

[4] Nielsen, O. A. and Jørgensen, R. M. Map-matching algorithms for GPS data - Methodology and test on data from the AKTA road pricing experiment in Copenhagen. World Conference on Transport Research Society (WCTRS), Proceedings, Paper 1435, D05 July 7th, Istanbul, July 4-8, 2004.

[5] Zabic, M., and Nielsen, O.A. A Geographic Information Analysis of Global Positioning System Quality for Road Pricing. (Submitted to Transportation Research Part C).

[6] A.B.O. Jensen, M. Zabic, H.M. Overø, B. Ravn, and O.A. Nielsen. Availability of GNSS for Road Pricing in Copenhagen. Proc. of the ION GNSS 2005 conference in Long Beach, CA, USA, 2005. 